\title{
Prototyping chips in minutes: Direct Laser Plotting (DLP) of functional microfluidic structures
}

\author{
Limu Wang ${ }^{a}$, Rimantas Kodzius ${ }^{b, d}$, Xin Yi ${ }^{a}$, Shunbo Li $^{c}$, Yu Sanna Hui ${ }^{a}$, Weijia Wen ${ }^{a}, b, c, *$

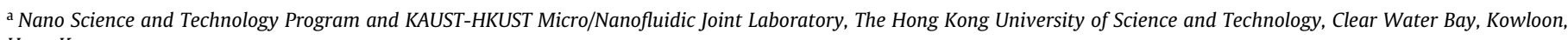 \\ Hong Kong \\ b KAUST-HKUST Micro/Nanofluidics Joint Laboratory, The Hong Kong University of Science and Technology, Clear Water Bay, Hong Kong \\ ${ }^{c}$ Department of Physics, The Hong Kong University of Science and Technology, Clear Water Bay, Kowloon, Hong Kong \\ ${ }^{\mathrm{d}}$ King Abdullah University of Science and Technology (KAUST), Physical Sciences and Engineering Division, Thuwal 23955-6900, Saudi Arabia
}

\section{A R T I C L E I N F O}

\section{Article history:}

Received 3 February 2012

Received in revised form 26 March 2012

Accepted 2 April 2012

Available online $\mathrm{xxx}$

\section{Keywords:}

Rapid chip prototyping

Direct Laser Plotting

PDMS

Microfluidic

Lab-On-a-Chip (LOC)

\begin{abstract}
A B S T R A C T
We report a fast and simple prototyping method to fabricate polymer-based microfluidic chips using Direct Laser Plotting (DLP) technique, by which various functional micro-structures can be realized within minutes, in a mask-free and out-of-cleanroom fashion. A 2D Computer-Aid-Design (CAD) software was employed to layout the required micro-structures and micro-channels, a $\mathrm{CO}_{2}$ laser plotter was then used to construct the microstructures. The desired patterns can be plotted directly on PDMS substrates and bio-compatible polymer films by manipulating the strength and density of laser pulses. With the DLP technique, chip-embedded micro-electrodes, micro-mixers and 3D microfluidic chips with 5 layers, which normally require several days of work in a cleanroom facility, can be fabricated in minutes in common laboratory. This novel method can produce microfluidic channels with average feature size of $100 \mu \mathrm{m}$, while feature size of $50 \mu \mathrm{m}$ or smaller is achievable by making use of the interference effect from laser impulsion. In this report, we present the optimized parameters for successful fabrication of 3D microchannels, micro-mixers and microfluidic chips for protein concentration measurements (Bovine Serum Albumine (BSA) test), and a novel procedure to pattern flexible embedding electrodes on PDMS-based microfluidic chips. DLP offers a convenient and low cost alternative to conventional microfluidic channel fabrication technique which relies on complicated and hazardous soft lithography process.
\end{abstract}

(c) 2012 Elsevier B.V. All rights reserved.

\section{Introduction}

Lab-On-a-Chip (LOC) system, which is an integration of several functional microfluidic devices, is highly desirable in microsynthesis and rapid diagnosis research because it comprises of different microfluidic devices packaged on a single chip of several square-centimeters in size [1]. LOC system offers convenience, portability, disposability, and integrability to tackle multiple problems in chemical, medical and biological perspectives simultaneously. Among the available building materials for LOC systems, polydimethylsiloxane (PDMS) [1,3] is the most

Abbreviations: BSA, Bovine Serum Albumine; DLP, Direct Laser Plotting; LOC, Lab-On-a-Chip; PDMS, polydimethylsiloxane; XPS, X-ray photoelectron spectroscopy.

* Corresponding author at: Department of Physics, The Hong Kong University of Science and Technology, Clear Water Bay, Kowloon, Hong Kong. Tel.: +852 23587979; fax: +852 23581652.

E-mail address: phwen@ust.hk (W.Wen). popular because of its advantageous attributes in elasticity, transparency, bio-compatibility, fabrication, and material cost. Conventionally, PDMS-based microfluidic chips are fabricated by soft-lithography, which entail standard lithography processes for fabricating microfluidic channels. Despite the fact that fine structures can be readily achievable by conventional soft lithography, the process itself is generally regarded as an expensive and time-consuming method because it demands for cleanroom environment and expensive instrumentation [1]. Conventional soft lithography will gradually become a limiting factor to the advancement of fabricating LOC systems.

Motivated by the growing demand for inexpensive and versatile microfluidic chips and LOC systems, recent research effort has been focusing on seeking alternatives to conventional cleanroombased soft lithography process. One such effort involves replacing expensive ultra-violet (UV) exposure machine with relatively inexpensive light sources, such as sunlight [4], or laser source from confocal microscope $[5,6]$. Other researchers investigate the issue from material selection perspective by replacing PDMS with ordinary printing paper as substrate material [7-9]. Despite of the 
efforts, paper substrate possesses fundamental limitations which prevent it from successfully substituting PDMS as substrate materials because the cellulous fiber in paper reacts with liquid molecules via absorption. Recently, attention has been drawn to laser ablation technique which produces microfluidic channels with reasonably good resolution [10-12]. Few other studies have been focusing on laser-plotted replica-molds for PDMS-based microchannels [13]. In fact, much efforts have been made to utilize direct laser ablation and casting on different alloy and polymer surfaces [14-17], to form microfluidic channels, large-scale integrated microstructures such as 3-dimensional (3D) photonic crystals [18] or microfluidic interlaying connections $[19,20]$. In this article, we present a comprehensive study on the fabrication of PDMS-based microfluidic chips and the embedded flexible microelectrodes using Direct Laser Plotting (DLP) technique.

We hereby reported a rapid fabrication technique for microfluidic channels, microstructures and flexible electrodes using DLP. DLP can dramatically reduce the fabrication time and complexity for microstructures. For example, fabricating microelectrodes which require a typical three days of work in a cleanroom facility can be dramatically reduced to a $30-\mathrm{min}$ process conducted in a common laboratory by adopting DLP. A micro-mixer can also be constructed easily by DLP in about $5 \mathrm{~min}$ with microchannels of the same quality and resolution as those fabricated by soft lithography process. Moreover, fabrication of PDMS-embedding-electrodes is generally regarded as a challenge in soft lithography because of the process complexity. However, this is not the case in DLP because the fabrication time for PDMS-embedding electrodes is less than $20 \mathrm{~min}$ in out-of-clean-room environment [21,22]. On the other hand, our study on DLP technique has extended to adhesive bio-compatible tapes and results have proven that $3 \mathrm{D}$ microfludic chips can be constructed on adhesive tapes within $10 \mathrm{~min}$. The motivation for adapting adhesive bio-compatible tapes is that bulk material naturally eliminates issues associated with sealing, and the need for half-curing and plasma-bonding. The typical feature size achieved by our laser plotter machine is around $100 \mu \mathrm{m}$, limited by the laser beam diameter. In fact, smaller feature size is readily achievable with advancement in laser plotter with higher resolution. Overall, our DLP technique offers advantages on time- and cost-saving advantages over conventional soft lithography by eliminating clean-room facility and complex fabrication steps.

\section{Materials and experiments}

\subsection{Materials and equipments}

PDMS (Sylgard 184, Dow Coring), bio-compatible adhesive tape (BIO-RAD, Microseal ${ }^{\circledR} \mathrm{B}$, Adhesive Sealer), silver power $(1.2-2.2 \mu \mathrm{m}$ silver platelet, Unist Business Corp., Shanghai), carbon power (40-100 nm carbon black power, Vulcan XC72-R, Cabot Inc.) a laser printer (Versa Laser System, model VR3.50, Universal Laser Systems, Ltd.), an ultrasonic system (1210, BRANSON), a scanning electron microscope (SEM, JSM-6300), a 3D microscope (VHX1000 , Keyence Corp.), laser-computer interface software (AutoCAD, Autodesk Inc., USA; CorelDRAW ${ }^{\circledR}$, Corel Corp.) and a spectrophotometer (Lambda 20 UV/VIS, Perkin Elmer).

The laser plotter employed in our experiments is a Model VR3.50 $\mathrm{CO}_{2}$ laser system, produced by Versa Laser System, Universal Laser Systems, Ltd. It has a maximum power of $25 \mathrm{~W}$, maximum pulse per inch (PPI) of 1000 , and laser moving speed ranging from $0.25 \mathrm{~mm} / \mathrm{s}$ to $25 \mathrm{~mm} / \mathrm{s}$. The laser plotter is connected to an in-house built exhaust system to prevent poisonous gas leakage and maintain a dust-free plotting environment for the laser.

\subsection{Material preparation}

PDMS bulk material: pre-polymer and PDMS curing agent were premixed at a weight ratio of 10:1. The mixture was poured into a flat flask and cured at $60^{\circ} \mathrm{C}$ for $2 \mathrm{~h}$.

Conductive AgPDMS/CPDMS gel: for the ease of structure patterning, PDMS pre-polymer for conductive gel application was diluted by heptane in a weight ratio of $1: 1$. The diluted PDMS prepolymer was mixed with the treated silver powder (for AgPDMS) or carbon black powder (for CPDMS) directly in a miller, in weight concentration 4:1 and 2:1 respectively [22].

A Bradford protein assay was used to verify the biocompatibility of the laser-plotted microfluidic chip. The experimental dye reagent concentrate solution (Thermo Fisher Scientific) contained 25\% Coomassie Brilliant Blue G-250 dye, 50\% phosphoric acid and 25\% methanol. The BSA dilution series (1500, 1250, 1000, 750,500 , and $250 \mu \mathrm{g} / \mathrm{ml}$ ) and the blank were prepared in a $1 \times$ synthetic urine solution containing $14.1 \mathrm{~g} \mathrm{NaCl}, 2.8 \mathrm{~g} \mathrm{KCl}, 17.3 \mathrm{~g}$ urea, $1.9 \mathrm{ml}$ of $25 \%, \mathrm{v} / \mathrm{v}$ ammonia solution, $0.6 \mathrm{~g} \mathrm{CaCl}_{2}$, and $0.43 \mathrm{~g} \mathrm{MgSO}_{4}$, filled to $1 \mathrm{~L}$ with the addition of $0.02 \mathrm{M} \mathrm{HCl}$ solution, for a final $\mathrm{pH}$ of 7.0.

\subsection{Fabrication}

The cured, flat PDMS bulk material was positioned on the metal mesh of the laser plotting system (see Supplementary information). The pattern to be plotted was designed using ordinary CAD software. Four parameters can be adjusted to create diversified patterns: laser power, pulse density (pulses per inch - PPI), focus and laser moving speed. In the fabrication process of PDMS-based microfluidic chips, a layer of plotted micro-patterns can easily be bonded to another layer by half-curing or plasma-bonding method. The same protocol can be applied to adhesive thin film or other type of thin film. By peeling off the protective side of the laser-plotted adhesive thin film, it can be adhered directly to a suitable substrate to form microfluidic channels or chips.

\subsection{Characterization}

To study the influence of the laser parameters on the microstructural configuration, the laser-plotted microstructures were examined under both optical microscopy and scanning electron microscopy (SEM). Prior to this, the plotted PDMS patterns were incubated for $10 \mathrm{~min}$ in ethanol (75\%) by ultrasonic cleaning, and then dried at $60^{\circ} \mathrm{C}$ for $2 \mathrm{~h}$. Prior to the SEM imaging, the samples were sputter-coated with gold. X-ray photoelectron spectroscopy (XPS) was employed for chemical composition analysis before and after laser plotting, while the Bradford assay method for the determination of protein concentrations in urine samples was utilized to test the function of microstructure and to verify the enzymatic compatibility of the laser-plotted PDMS.

\section{Results and discussion}

\subsection{Influence of laser parameters}

At the beginning of our study, we realized that the channel feature size is the key factor constraining the chip fabrication by DLP. Microstructures constructed by DLP are based upon thermal degradation effect, therefore the plotted PDMS-patterns show feature size of $100 \mu \mathrm{m}$. There are 3 crucial parameters governing the settings of laser machines: laser power, moving speed, and pulses per inch (PPI), which will affect the final profile of microchannels. In order to investigate the effects of these parameters on laser-plotted microfluidic channels, we examined both the chemical composition and geometry of the plotted microstructures on PDMS, and 

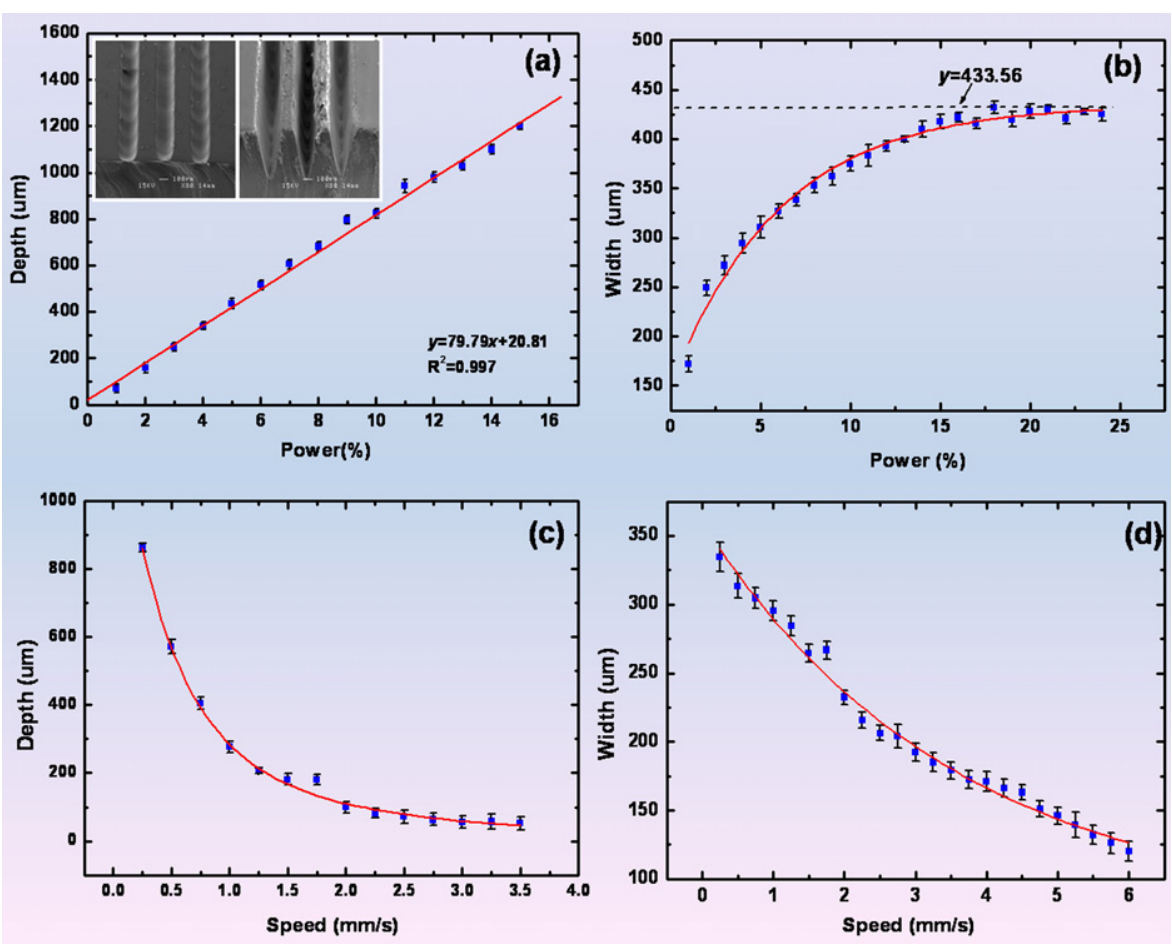

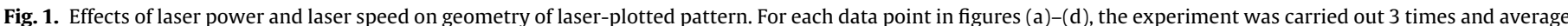

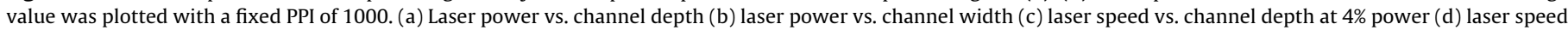
vs. channel width at $4 \%$ power.

obtained the optimized laser power and speed settings for direct prototyping of microfluidic chips with specific applications. The same experiment was carried out three times for each data point and the average values were plotted.

\subsubsection{Laser power and laser speed}

Laser power has two main effects on PDMS substrate: changing the feature size of plotted PDMS-based microchannel; changing the chemical composition after laser degradation. The laser moving speed defines the heating time of the substrate surface as it is carved by the laser beam, which in turn affects the degradation profile. The calibration was performed by plotting straight lines at 1000 PPI with increasing power and speed, the results were then measured under microscope. The left inset of Fig. 1(a) shows the result for $1 \%$ power (i.e. $0.25 \mathrm{~W}$ ), and the right one, that for $5 \%$ (i.e. $1.25 \mathrm{~W})$. It was found that when the laser power was increased beyond $5 \%$, the plotted PDMS surface and channel edge became rough and dusty, and were consistent with the chemical composition analysis results. To achieve a suitable depth for microfluidic application, the laser power was limited to $1-3 \%$ of its full range, which is $25 \mathrm{~W}$. It was found that the laser speed affects the edge roughness and the amount of dust formed. The amount of dust formed was reduced at a higher laser speed. We have optimized the laser speed to be $0.75 \mathrm{~mm} / \mathrm{s}$, at which the line depth increased linearly with the laser power, which is a very favorable property for designing laser plotting templates. Therefore, the laser speed was fixed for the study on power dependence of the laser. Meanwhile, the laser plotted line width increase in sigmoid fashion and an asymptote was observed when the laser power was higher than 15\%: under these conditions, the channels tend to approach and maintain a constant width of $\sim 430 \mu \mathrm{m}$ (Fig. 1b), which was consistent with PDMS degradation property. The channel depth and width have an approximately reciprocal relation to the laser's speed (Fig. 1c and d) with a fixed power of $4 \%$. The experimental results have shown a single-valued correlation between the laser parameters and the channel geometry. The correlation equation provides the essential information on the design of microchannels for specific microfluidic applications.

The second experimental set was designed to study the dot profile under different laser powers (Fig. $2 \mathrm{~g}-\mathrm{i}$ are enlarged images from Fig. 2d-f). Maintaining the same PPI (75) and laser speed $(0.75 \mathrm{~mm} / \mathrm{s})$, it is clear that the dot depth and profile were influenced significantly by the laser power (1\%,3\%, $5 \%)$ : the dots became deeper in depth and sharper at the edge as the power increased. The power condition in Fig. $2 \mathrm{f}$ and e was large enough to penetrate a PDMS sheet of $500 \mu \mathrm{m}$ thickness and form funnel arrays in which each funnel has an upper diameter of $300 \mu \mathrm{m}$ and a lower diameter of 30-50 $\mu \mathrm{m}$. Such funnel arrays are very useful in cell screening process, formation of microspheres in microfluidic chips, and in micro-sized emulsion application. In particular, multiple micro-well arrays can be fabricated in a single run which are ideal miniature experimental platforms for high throughout drug screening and chemical analysis with nano/pico dosage.

\subsubsection{Laser beam density}

Laser plotting is executed by a continuous laser beam composed of rapidly moving high-density laser pulses that determine the spatial printing resolution. Pulses per inch (PPI), a measure as well as a parameter, is a correlation between printing quality and laser beam density. In addition to smooth fluidic channels, laser plotting technique can achieve unique architectures such as cone or funnel shapes that are considerably time consuming in conventional soft lithography method. Therefore we conducted thorough tests in order to understand how PPI correlates with other parameters and its effect on pattern feature and geometry. The results are presented in Fig. 2. The laser employed in this experiment can achieve PPI as high as 1000 (i.e. 1000 pulses per inch). We performed an initial set of evaluations (Fig. $2 \mathrm{a}-\mathrm{c}$ ) to evaluate the pattern quality under different PPIs. The result of highest value of PPI which produced the smoothest line on PDMS substrate (power $1 \%$, speed $0.75 \mathrm{~mm} / \mathrm{s}$, 

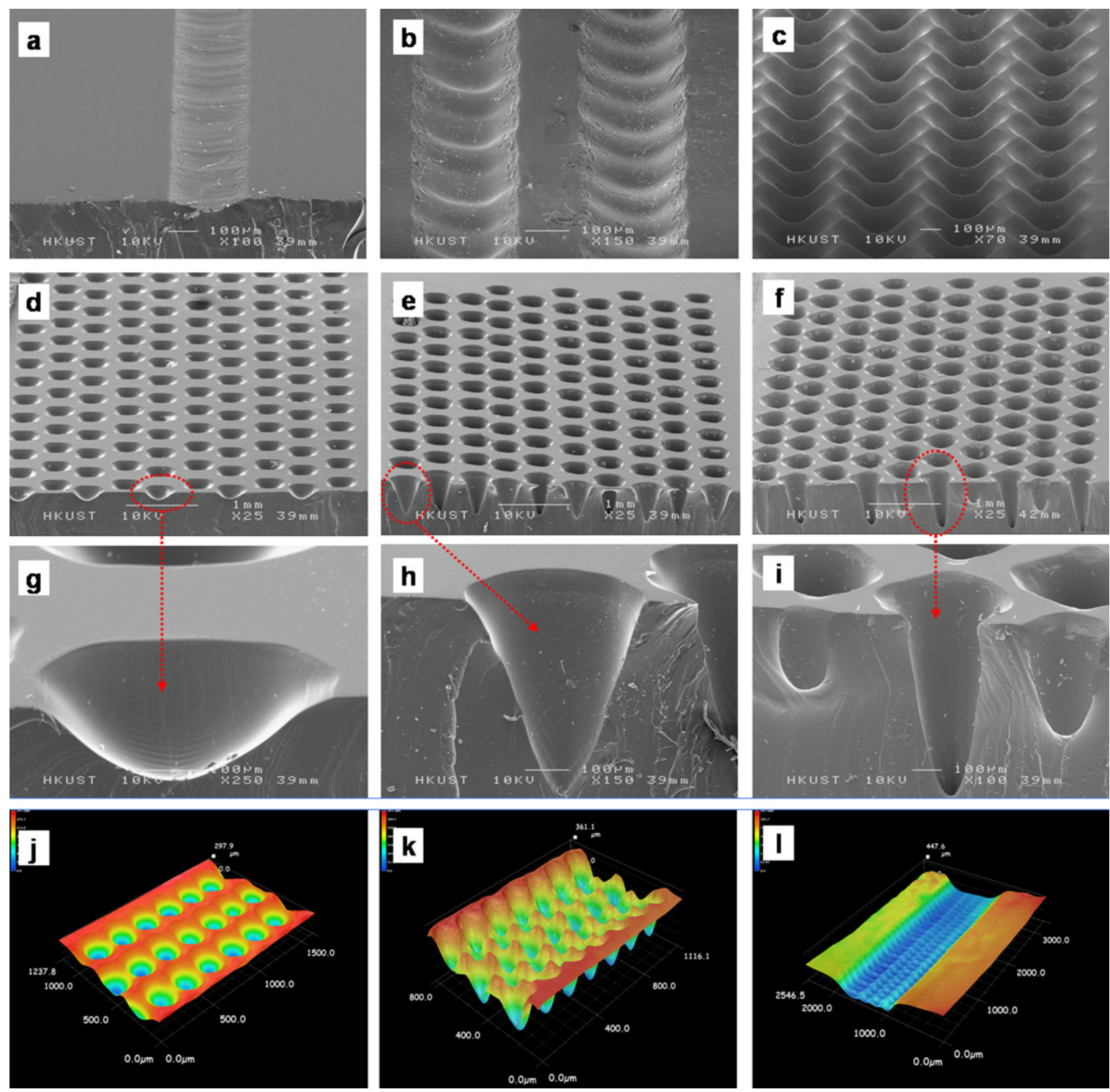

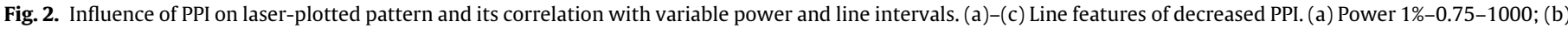

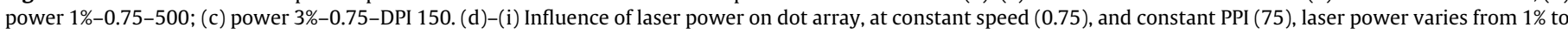

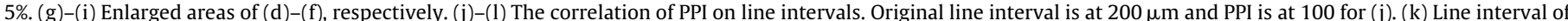

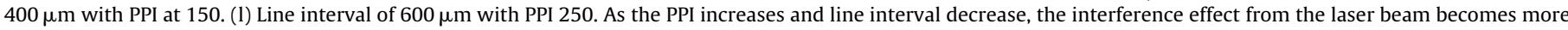
dominant.

1000 PPI; shortened hereafter to the [power]\%-[speed]-[PPI] format for ease of expression) can be seen in Fig. 2a. When PPI was lowered to 500 (Fig. 2b), the bottom surface became uneven. Lowering the PPI further to 150 (3\%-0.75-150), as shown in Fig. 2c, it is obvious that a line was composed of a stream of dots, and further lowering PPI led to orderly sets of dots. The uniform dot array forming 4 lines as shown in Fig. 2c reflects the optimized value of PPI with controlled line spacing.

During the PPI-optimization process, we discovered an interesting effect when manipulating the distance between two lines (or dot streams). The interference effect due to thermal degradation of adjacent dot streams has created distinctive micro-patterns which can be useful in advanced microfluidic chips. For example, a micro-mixer is a key component in microfluidic chemistry, which calls for two-step soft-lithography patterning in conventional soft-lithography but a single run of DLP. These interference patterns open the doors of alternatives to micro-fabrication process for many microfluidic applications.
The third set of experiments focuses on the correlation of line interval and PPI. It can be seen in Fig. $2 c$ that under the condition of $3 \%-0.75-150$, in addition to thermal degradation interference between dots in the same stream, there is also similar interference between adjacent streams when the line interval is smaller than the dot width. The experimental parameters were 3\%-0.75-100 as shown in Fig. 2j, in which no interference was observed between adjacent dots or lines. In Fig. $2 \mathrm{j}$ and 1 , the line interval decreased by $200 \mu \mathrm{m}$, while the PPI increased by 50 and 150, respectively. A stronger interference was observed as the PPI increased and line interval decreased. Taking Fig. $2 \mathrm{j}$ as an example, the original interval separating two dots, which preserved the same height as the nonplotted area, shrank to little hill tops when the PPI increased and line separation decreased (Fig. 2k), and disappeared when PPI and line interval were further adjusted (Fig. 2l).

\subsubsection{Chemical composition analysis}

Laser plotting on polymer substrate are based on thermal degradation effect. For PDMS, it undergoes depolymerization in the 


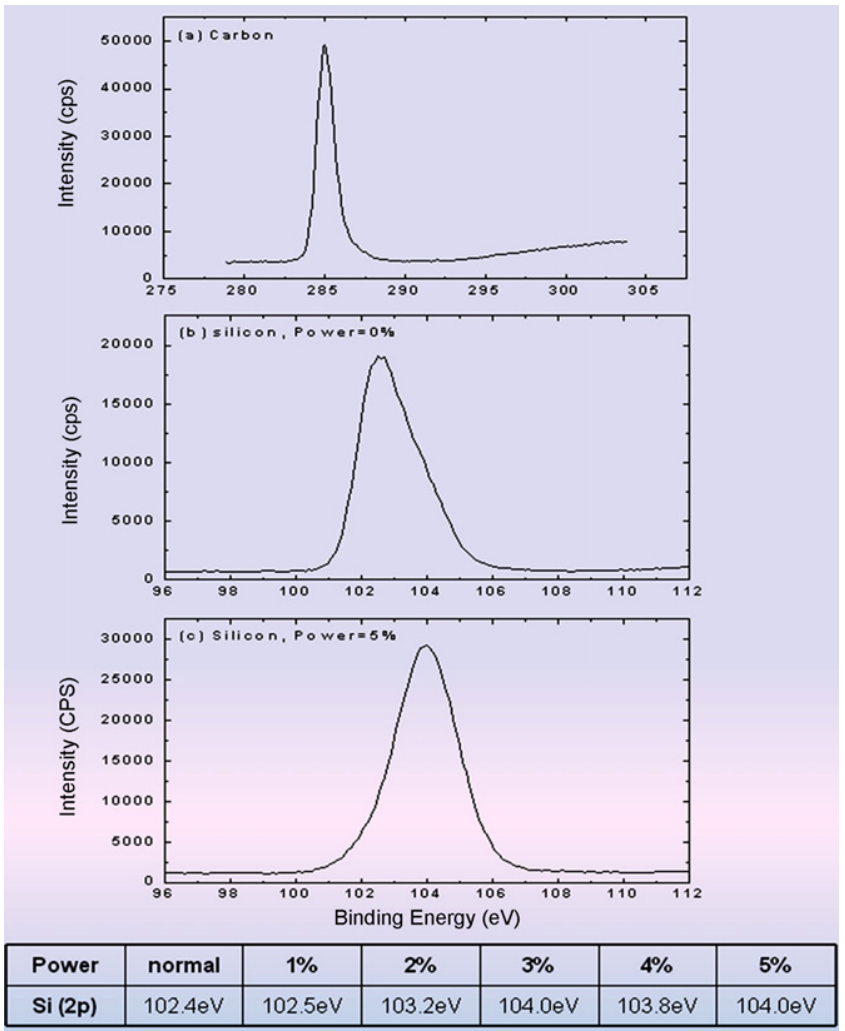

Fig. 3. X-ray photoelectron spectroscopy (XPS) result for chemical composition analysis. Detailed binding energy for (a) carbon bonding energy, as the reference. (b) Binding energy of silicon for bulk PDMS material is $102.4 \mathrm{eV}$. (c) Binding energy of silicon for plotted PDMS by DLP at $5 \%$ power is $104.0 \mathrm{eV}$, which is the binding energy of $\mathrm{SiO}_{2}$. The change of silicon's binding energy of plotted PDMS by DLP at $1-5 \%$ power is presented in the table at the bottom, which clearly indicates that the amount of $\mathrm{SiO}_{2}$ increases as the plotting power increases.

400-650 ${ }^{\circ} \mathrm{C}$ temperature range, resulting in cyclic oligomers. The formula for PDMS is $\left[\mathrm{Si}\left(\mathrm{CH}_{3}\right)_{2} \mathrm{O}\right]_{n}$, suggesting that it decomposes into small molecules including $\mathrm{SiO}_{2}$ and $\mathrm{CO}_{2}$, which was in fact proved by the XPS results as shown in Fig. 3. Oxygen and silicon both show movement to higher binding energy. The binding energy of $\mathrm{Si}$ in the $-(\mathrm{Si}-\mathrm{O}-\mathrm{Si})-$ structure is $102.4 \mathrm{eV}$, while in $\mathrm{SiO}_{2}$ it is $\sim 104 \mathrm{eV}$, which clearly shows that, for the same laser plotting structure, a higher laser energy produces more $\mathrm{SiO}_{2}$ on the substrate surface. It was found that when the laser power is higher than $5 \%$, a white patch of dust deposition accumulated on the PDMS surface, but almost no dust accumulation when the power was lower than $2 \%$, which was consistent with the XPS result. On the other hand, it is recommended, for high-power laser-plotting process, the PDMS surface should be rinsed prior to bonding in order to increase the bonding stress and eliminate fluidic channel blockage.

\subsection{Fast fabrication of functional microfluidic chips}

Practical applications of DLP in microfluidic research include the formation of various microstructures in a convenient and fast paced setting which is especially useful in the integration of different functional microfluidic components. In this report, 3D microfluidic chips, mixers with ribcage structure, and fast patterning of embedding electrodes on PDMS chips are excellent example of successful fabrication based on optimized laser parameter settings. The fabrication process of each of the aforementioned example can be accomplished within 10 min with an ingenious CAD design (see Supplementary information). In our experiment, we have achieved microchannels with about $100 \mu \mathrm{m}$ in diameter with feature size of about $50 \mu \mathrm{m}$. We fabricated three samples per design with measured consistence in spatial parameters. The minimal controllable etching depth of $100 \mu \mathrm{m}$ could be achieved by adjusting the laser power by $0.5 \%$ according to our data in Fig. 1(a). It is believed that smaller channel depth is achievable by using a laser source with lower maximum laser power because the power step size can be smaller for better precision and controllability.

\subsection{Mask-free rapid 3D chip fabrication}

The fabrication of laser-plotted thin film for microfluidic applications has recently been reported by other researchers $[17,18,23,24]$. However, the growing demand for ever-advanced microfluidic structures raises the question of how to integrate microfluidic channels into thin films. Wen's group has applied laser plotter in wax bonded polymer and glued paper chips $[8,10]$, in a layer by layer fashion. Here we introduce a kind of self-sealing bio-compatible adhesive polymer tape as the alternative to manual sealing of polymer by external sealing chemicals which requires extra fabrication step. As implied by its name, bio-compatible adhesive tape possesses both biocompatibility and glutinosity, which their dual attributes help to simplify the 3D chip fabrication process in laser plotting and alignment. The process can be accomplished within 10 min, as demonstrated in Fig. 4 . The adhesive side of the bio-compatible tape is protected by smooth yellow paper before bonding, which undergoes DLP to create the desired pattern. After peeling off the protective yellow paper, the plotted thin film can be directly bonded to another layer of thin film. The process can be repeated indefinitely to form multi-layered chip. For 3D chips with $\mathrm{n}$ layers of channels, a $(n-1)$ fluid-connecting layer is needed. In order to demonstrate the incredibly simple and fast 3D chip fabrication, we considered the typical cross-channel architecture as an example; a brief summary of the design and fabrication details is provided in Fig. 4. Fluid channels in separate layers are plotted in layer 1 and layer 3, while the connecting port is plotted in layer 2 . The 3 layers are carefully patterned, aligned and bonded to each other after the removal of the protective yellow paper, and finally sealed by 2 blank layers from each side, thereby accomplishing the well-known "jumped line" crossing channel. Excluding the time spent in designing the 3-layer pattern on CAD, the laser plotter takes no more than $3 \mathrm{~min}$ to imprint the patterns on the substrate, while the layer bonding process requires only 2-5 min each.

\subsection{Micro-concentration separator integrated with micro-mixer}

By creatively incorporating laser-plotted lines and dots as the basic building blocks for functional microfluidic chips, such as mixing structure with integrated concentration separator, the fabrication process can be realized by a mask-free laser plotting process completed in a single run. Patterned bottom surface of microfluidic channel can form effective microfluidic mixer, as proved by the well-known fishbone mixing structure [25], a 3D bottom channel surface that provides turbulence to streams. This kind of structure calls for double layer SU8 masks, which may take several hours to several days to fabricate, and has high requirement for fine alignment between repetitive UV exposures. Here we integrated a ribcage structure, similar to fishbone structure into concentration separator, and were able to finish the fabrication by a single run DLP within $10 \mathrm{~min}$. We were able to demonstrate that the ribcage structure was as effective as the reported fishbone structure [25] and it functioned well with the integrated concentration separator Fig. 5, which is a successful example of functional micro-mixer.

The micro-mixer design was initiated with a CAD drawing with single layer pattern, and each fluidic channel appeared as a single continuous line for the laser plotter (see Supplementary 


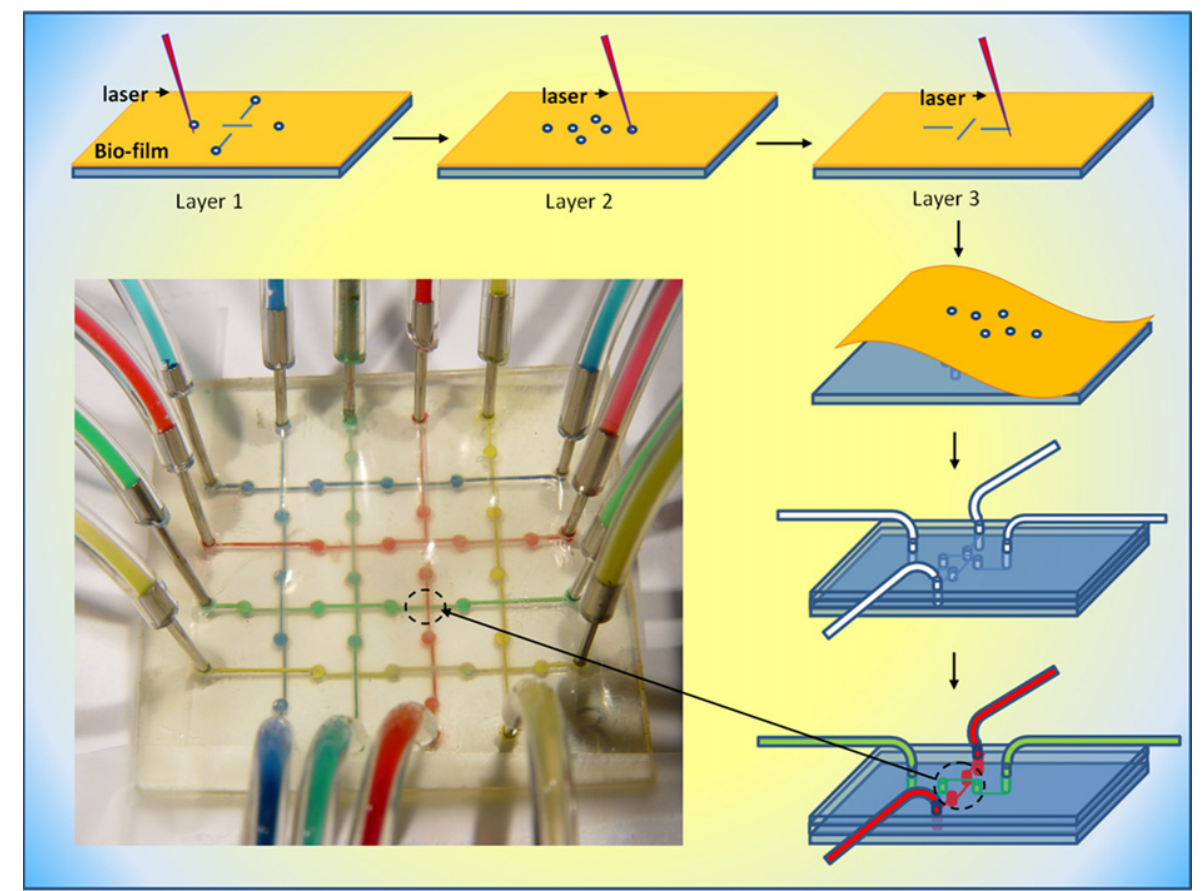

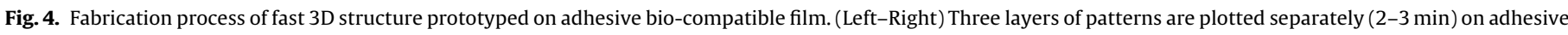
thin film, the protective paper is peeled off, each layer is sequentially aligned and bonded (5-6 min), to form 3D chip.

information). The smooth channel surface and the ribcage structure are distinguished by different colors in software, according to which the laser plotter specifies different power and PPI. For example, the smooth surfaces, regardless of straight lines or curves (area 1 in Fig. 5a), are marked by black lines, which indicates 1000 PPI, while the ribcage structure (area 2 in Fig. 5b), that is, the low-PPI structure, is marked by red, indicating 110 PPI. Then, the file was sent to laser plotter, to print the entire structure in a single run. The mixing efficiency was verified by two-stream mixing experiments (Fig. 5c), in which two colored streams (flow rate: $0.05 \mathrm{ml} / \mathrm{h}$ ) were
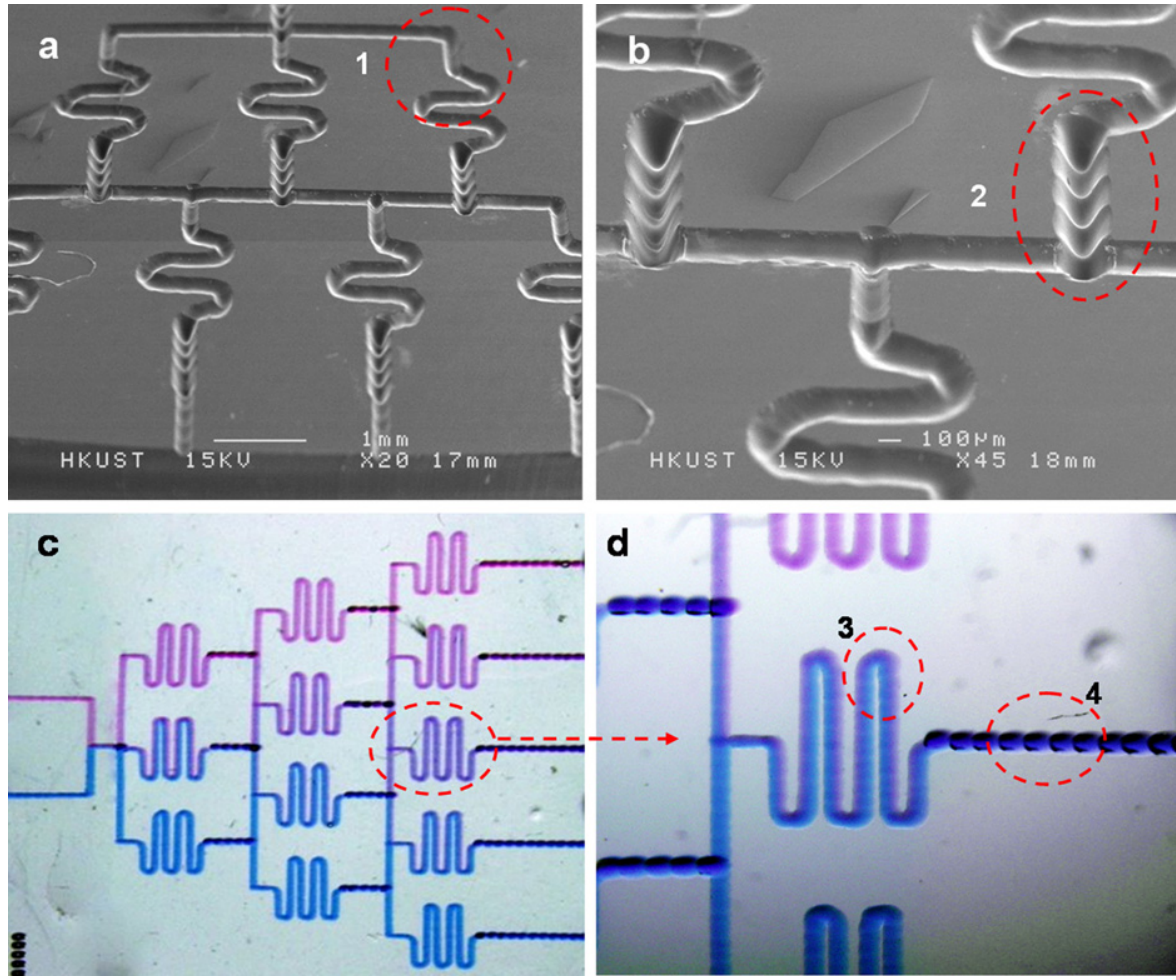

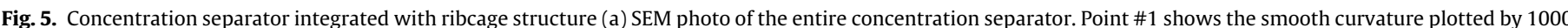

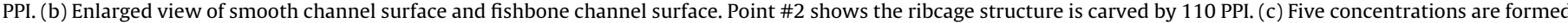

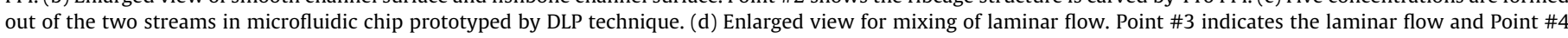
shows the result of mixing within the ribcage structure. 
a

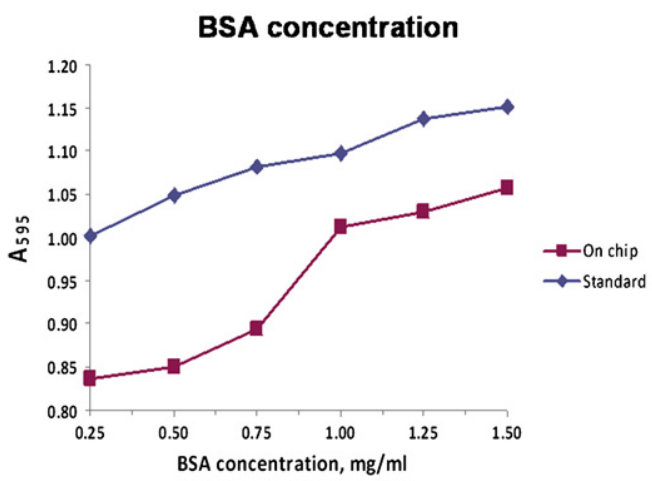

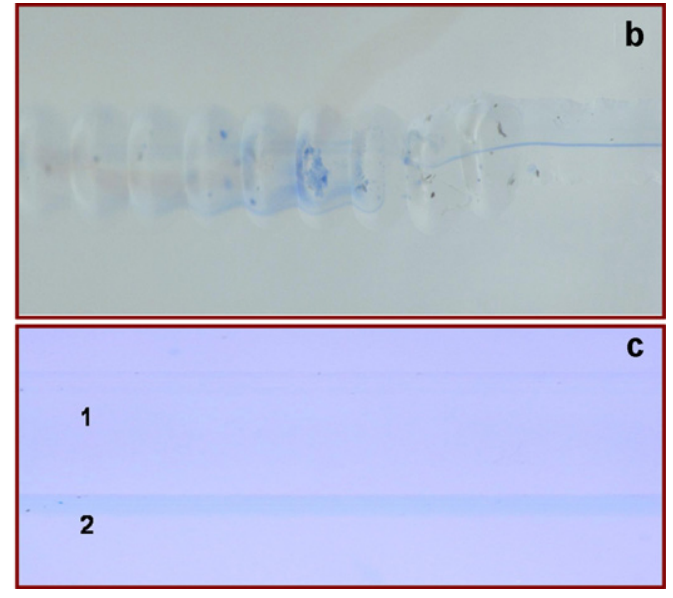

b

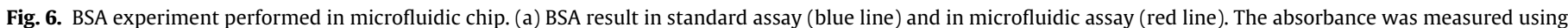

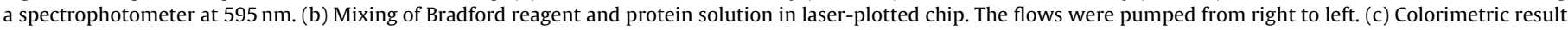

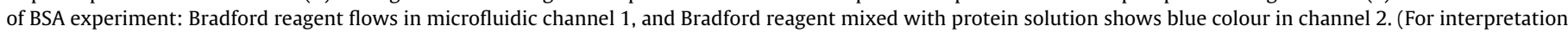
of the references to color in this figure legend, the reader is referred to the web version of the article.)

mixed in five different concentrations. An enlarged view (Fig. 5d) shows that the two streams formed a laminar flow before entering the ribcage structure (area 3 in Fig. 5d), and mixed immediately after two turns into the ribcage structure (area 4 in Fig. 5d).

Application of aforementioned fast patterning of mixing components lies in microfluidic synthesis or biochemical reactions, such as Bradford assay method (BSA experiment) for determination of protein concentrations in urine samples, which is a fast method that involves only a mixing step and yields a stable colorimetric response. It is based on the color change of the Coomassie Brilliant Blue G-250 dye (from 465 to $595 \mathrm{~nm}$ ) in response to various concentrations of protein. The diluted BSA solution samples were separately mixed at a $1: 1$ volume ratio with Bradford reagent. A typical Bradford assay experimental result was provided in Fig. 6a, in blue. The absorbance of the mixed solutions reacted in both conventional tube and in the laser-plotted PDMS microchannels, were measured at $595 \mathrm{~nm}$. It is clear that the absorbance increases when the protein concentration increases. The Fig. 6 results show that the absorbance readings taken from the solutions mixed in the laser-plotted chip were a little smaller than those taken from the solutions mixed in the conventional tube, because of the low adsorption of BSA on the chip walls. Fig. 6b shows the reagent mixing process in micro-mixer with ribcage structure. The BSA results can be visually monitored, either by microscope or the naked eye, as suggested by Fig. 6c.

\subsection{Rapid electrode patterning on PDMS microfluidic chip}

Electrodes are indispensable components in microfluidics, electrophoresis [26,27], signal detection [28], and droplet/fluid control $[29,30]$. Unlike sputtering microelectrodes on hard substrates such as silicon and glass, patterning microelectrodes on flexible plastic substrates such as PDMS has remained relatively challenging for nearly a decade. With DLP, one can pattern microstructures directly on PDMS. Together with the conductive composites, microchips with embedded electrodes can be readily fabricated using DLP in the matter of minutes. Fig. 7 illustrates the main processes of the rapid electrode patterning method. First, cured, flat bulk PDMS is seamlessly covered by a piece of clean weighing paper, which acts as a dust-free cover mold. Then, the laser is applied, carving a continuous electrode line where desired. AgPDMS gel or CPDMS gel is brushed onto that electrode line afterwards, with the weighing paper as a cover, to protect the rest of the bulk PDMS from the conductive gel. After the electrode line is filled by conductive paste, the whole pattern is let stand for 5 min for heptene evaporation, after which the weighing paper is carefully removed (Fig. 7b). As shown in Fig. 7b, the descending wall thickness (from $204 \mu \mathrm{m}$ to $104 \mu \mathrm{m}$ ) is the evidence of a successful tuning of line thickness by adjusting laser power. With optimized parameters, laser power can be adjusted to achieve various line widths to meet the chip design requirements. A second cut thereafter is made through the PDMS-embedded conductive line to divide it into two adjacent electrodes and simultaneously generate the fluidic channel that passes by these two electrodes (Fig. 7c). Fluidic inlets and outlets are drilled out while the microfluidic half-plane is ready to be bonded to the blank half to form a complete microfluidic chip with embedded microelectrodes. Fig. 7c shows the result with AgPDMS, while Fig. 7d shows that with CPDMS.

The time-efficient 5-step electrode fabrication process can be briefly outlined as follows: (1) affix the shielding paper, (2) plot the electrode channel, (3) fill the conductive composite, (4) remove the paper, (5) plot the fluidic channel. The whole process takes less than $10 \mathrm{~min}$ for a $10 \mathrm{~cm} \times 10 \mathrm{~cm}$ chip. The electrode is tested by a microfluidic signal generated by a conductive droplet (saturated $\mathrm{KCl}$ solution, $20^{\circ} \mathrm{C}$ ) carried by insulate fluid (100 cSt silicone oil). As shown in Fig. 8a, the conductive droplet is produced by a flow-focusing structure upstream: when the droplets pass by the AgPDMS electrode pair, there is a significant change in the voltage share, which is recorded by computer (Fig. 8b). This method is the simplest and fastest method to fabricate PDMS-chip-embedded electrodes, and makes feasible, moreover, large-scale complex structures.

\subsection{Fast fabrication technique}

Large amount of prototyping microfluidic chips is consumed during testing and characterization stage. Conventional chip fabrication process, such as soft lithography, is often very costly and time-consuming especially during the early stage of the research. The DLP, in contrary, provides a low-cost and time-saving solution to the fabrication of microfluidic chips. Chip fabrication is considered as the most time consuming step in microfluidic research. In particular, the SU8 process takes $0.5-1$ day to form microchannels. One more day is required for each additional photoresist pattern layer (see Supplementary information) if a chip-embedded electrode or 3D structure is desired. However, the soft lithography processes which demand high-resolution and high-cleanliness are generally found to be unnecessary in many microfluidic 


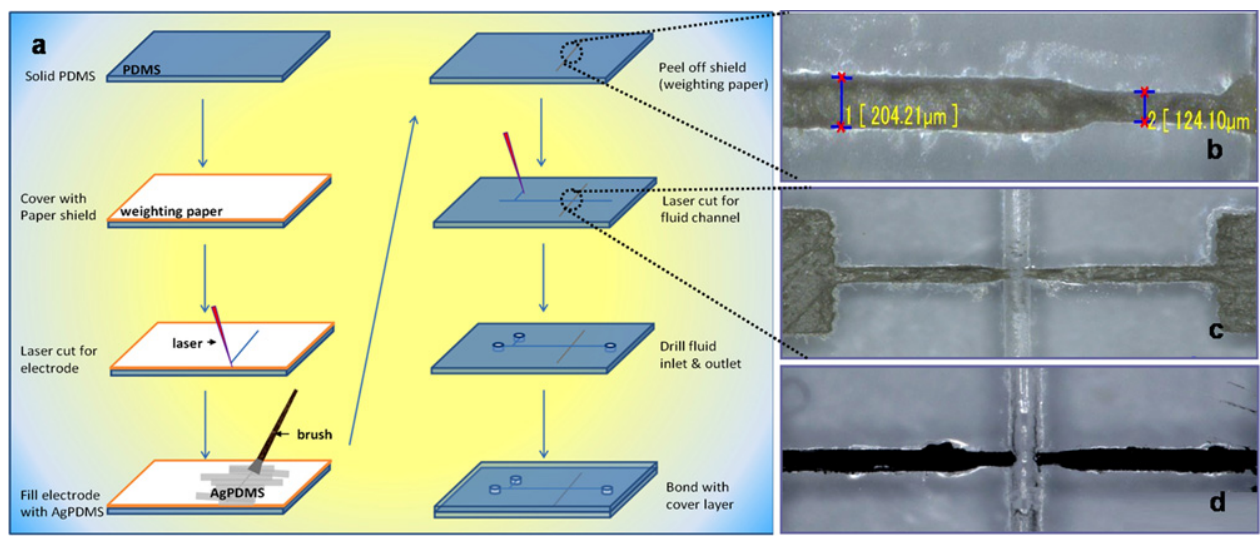

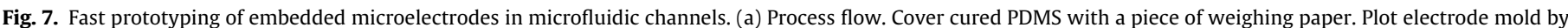

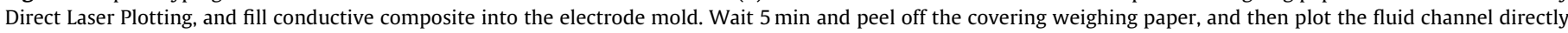

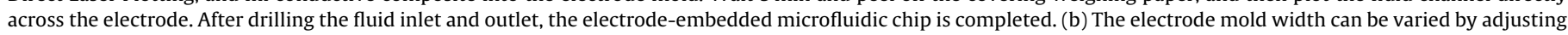
the laser power. (c) Top view of AgPDMS-electrodes-embedded microfluidic channel. (d) Top view of CPDMS-electrodes-embedded microfluidic channel.

(a)

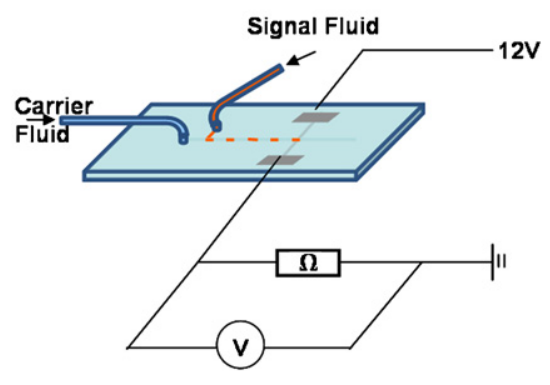

(b)

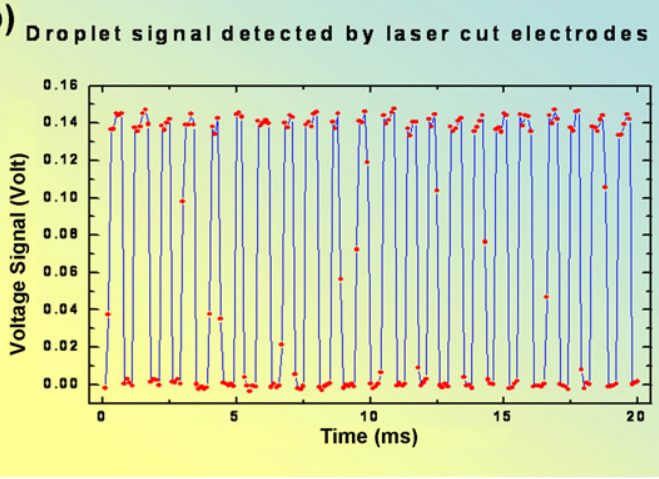

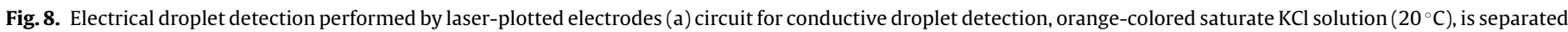
in the droplets by carrier fluid (100 cSt silicone oil) (b) electric signal detected by laser-plotted electrodes.

applications. Nonetheless, researchers have to bear the high equipment price, tedious procedures, and poisonous chemicals (such as HF and SU8 solvent) to fabricate a testing chip in $30 \mathrm{~h}$ which in contrary, take only $30 \mathrm{~min}$ to complete in DLP. Despite the fact that high-standard labs and clean-room environments are established for primary-stage microfluidic research, the environmental factor is fundamentally in contrary to the original goals of microfluidics development: inexpensive, convenience, portability, environmental friendly and disposability. Employing the DLP technique for microfluidic fabrication, a typical 3-day fabrication process is reduced nearly to an hour, leaving more time for researchers to verify chip function and improve fluidic design. The saved time and resources can be utilized more effectively on realizing new microfluidic functions.

\section{Conclusions}

DLP is a convenient and time-saving method for fabricating microfluidic devices. This method agrees with the attributes in microfluidic fabrication: convenience, inexpensiveness, biocompatibility and disposability. As an alternative to mold-replica fabrication process, DLP is a maskless method which allows one to plot micropatterns directly onto bulk material, forming complex microstructures and even microelectrode pairs. In comparison to conventional soft lithography, DLP eliminates the need for cleanroom facility and hazardous lithographic process. Most importantly, the material selection can be more diversified leading to enhancement in process compatibility. Moreover, the fabrication time can be dramatically reduced from days to few hours, and sometimes in minutes, saving resources for chip design and testing. In terms of instrumentation, DLP is advantageous over lithographic process because a single laser plotting machine is sufficient. Using DLP, we have successfully fabricated PDMS microchannels, micro-mixer and 3D microchips. Rapid fabrication of chip-embedded electrodes fabrication was also reported, which solve the challenge faced by conventional method of patterning electrode on PDMS, particularly when the material is not wetting with metal. Chemical composition analysis was also carried out to study the thermal degradation effect due to laser ablation. BSA experiments was performed to prove the enzymatic compatibility of laser plotted chips. By employing DLP, prototyping process of microfluidic chip has been dramatically simplified, endeavouring the fabrication of microfluidic chips by saving on cost, time and resource.

\section{Acknowledgements}

This publication is based on work partially supported by Award No. SA-C0040/UK-C0016, made by King Abdullah University of Science and Technology (KAUST), and Hong Kong RGC grants HKUST 603608 and 604710.

\section{Appendix A. Supplementary data}

Supplementary data associated with this article can be found, in the online version, at http://dx.doi.org/10.1016/j.snb.2012.04.011. 


\section{References}

[1] B.H. Weigl, R. Bardell, T. Schulte, F. Battrell, J. Hayenga, Towards non- and minimally instrumented, microfluidics-based diagnostic devices, Biomedical Microdevices 3 (2001) 267-274.

[3] M.P. Chang, M.M. Maharbiz, Electrostatically-driven elastomer components for user-reconfigurable high density microfluidics, Lab on a Chip 9 (2009) 1274-1281.

[4] J. Ma, L. Jiang, X. Pan, H. Ma, B. Lin, J. Qin, A simple photolithography method for microfluidic device fabrication using sunlight as UV source, Microfluidics and Nanofluidics 9 (2010) 1247-1252.

[5] J.S. Miller, M.I. Béthencourt, M. Hahn, T.R. Lee, J.L. West, Laser-scanning lithography (LSL) for the soft lithographic patterning of cell-adhesive self-assembled monolayers, Biotechnology and Bioengineering 93 (2006) 1060-1068.

[6] M.S. Hahn, J.S. Miller, J.L. West, Laser scanning lithography for surface micropatterning on hydrogels, Advanced Materials 17 (2005) 2939-2942.

[7] A.W. Martinez, S.T. Phillips, G.M. Whitesides, E. Carrilho, Diagnostics for the developing world: microfluidic paper-based analytical devices, Analytical Chemistry 82 (2010) 3-10.

[8] X. Yi, R. Kodzius, X. Gong, K. Xiao, W. Wen, A simple method of fabricating mask-free microfluidic devices for biological analysis, Biomicrofluidics 4 (2010) $1-8$.

[9] R. Lu, W. Shi, L. Jiang, J. Qin, B. Lin, Rapid prototyping of paper-based microfluidics with wax for low-cost, portable bioassay, Electrophoresis 30 (2009) 1497-1500.

[10] X. Gong, X. Yi, K. Xiao, S. Li, R. Kodzius, J. Qin, W. Wen, Wax-bonding 3D microfluidic chips, Lab on a Chip 10 (2010) 2622-2627.

[11] W. Wang, S. Zhao, T. Pan, Lab-on-a-print: from a single polymer film to threedimensional integrated microfluidics, Lab on a Chip 9 (2009) 1133-1137.

[12] D.A. Bruzewicz, M. Reches, G.M. Whitesides, Low-cost printing of PDMS barriers to define microchannels in paper, Analytical Chemistry 80 (2008) 3387-3392.

[13] G. Kumi, C.O. Yanez, K.D. Belfield, J.T. Fourkas, High-speed multuphoton absorption polymerization: fabrication of microfluidic channels with arbitrary cross-sections and high aspect ratios, Lab on a Chip 10 (2010) 1057-1060.

[14] C.Z. Dinu, V. Dinca, J. Howard, D.B. Chrisey, Printing technologies for fabrication of bioactive and regular microarrays of streptavidin, Applied Surface Science 253 (2007) 8119-8124.

[15] T. Hong, W. Ju, M. Wu, C. Tai, C. Tsai, L. Fu, Rapid prototyping of PMMA microfluidic chips utilizing a $\mathrm{CO}_{2}$ laser, Microfluidics and Nanofluidics 9(2010) 1125-1133.

[16] C.K. Chung, H.C. Chang, T.R. Shih, S.L. Lin, E.J. Hsiao, Y.S. Chen, E.C. Chang, C.C. Chen, C.C. Lin, Water-assisted $\mathrm{CO}_{2}$ laser ablated glass and modified thermal bonding for capillary-driven bio-fluidic application, Biomedical Microdevices 12 (2010) 107-114

[17] C.K. Malek, L. Robert, R. Salut, Femtosecond laser machining and lamination for large-area flexible organic microfluidic chips, EPJ Applied Physics 46 (2009) 12503-1-12503-5.

[18] M. Deubel, G. Von Freymann, M. Wegener, S. Pereira, K. Busch, C.M. Soukoulis, Direct laser writing of three-dimensional photonic-crystal templates for telecommunications, Nature Materials 3 (2004) 444-447.

[19] J. Huft, D.J. Da Costa, D. Walker, C.L. Hansen, Three-dimensional large-scale microfluidic integration by laser ablation of interlayer connections, Lab on a Chip 10 (2010) 2358-2365.

[20] S. Queste, R. Salut, S. Clatot, J. Rauch, C.G. Khan Malek, Manufacture of microfluidic glass chips by deep plasma etching, femtosecond laser ablation, and anodic bonding, Microsystem Technologies 16 (2010) 1485-1493.

[21] L. Wang, M. Zhang, J. Li, X. Gong, W. Wen, Logic control of microfluidics with smart colloid, Lab on a Chip 10 (2010) 2869-2874.

[22] X. Niu, S. Peng, L. Liu, W. Wen, P. Sheng, Characterizing and patterning of PDMSbased conducting composites, Advanced Materials 19 (2007) 2682.

[23] K. Conlisk, R. Sherlock, G.M. O'Connor, T.J. Glynn, Rapid prototyping process based on the use of an intelligent pinhole mask and $193 \mathrm{~nm}$ excimer laser used to fabricate polymer microfluidic devices, in: ICALEO 2009 - 28th International Congress on Applications of Lasers and Electro-Optics, Congress Proceedings, vol. 102, 2009.

[24] P. Nath, D. Fung, Y.A. Kunde, A. Zeytun, B. Branch, G. Goddard, Fabrication of a multichannel PDMS/glass analytical microsystem with integrated electrodes for amperometric detection, Lab on a Chip 10 (2010) 2286-2291.
[25] A.J. DeMello, Control and detection of chemical reactions in microfluidic systems, Nature 442 (2006) 394-402.

[26] B. Li, L. Jiang, Q. Wang, J. Qin, B. Lin, Micropumps actuated negative pressure injection for microchip electrophoresis, Electrophoresis 29 (2008) 4906-4913.

[27] Y. Luo, Q. Zhang, J. Qin, B. Lin, Further improvement of hydrostatic pressure sample injection for microchip electrophoresis, Electrophoresis 28 (2007) $4769-4771$.

[28] X. Niu, M. Zhang, S. Peng, W. Wen, P. Sheng, Real-time detection, control, and sorting of microfluidic droplets, Biomicrofluidics 1 (2007) 044101.

[29] X.X. Niu, M. Zhang, J. Wu, W. Wen, P. Sheng, Generation and manipulation of smart droplets, Soft Matter 5 (2009) 576-581.

[30] M. Zhang, J. Wu, X. Niu, W. Wen, P. Sheng, Manipulations of microfluidic droplets using electrorheological carrier fluid, Physical Review E 78 (2008) 066305.

\section{Biographies}

Limu Wang received her PhD from Nano Science and Technology Program of the Hong Kong University of Science and Technology, 2012. Her main research areas are microfluidics and LOC (Lab-On-a-Chip).

Rimantas Kodzius graduated with a PhD degree from the University of Salzburg (Austria, EU) with Prof. Reto Crameri (Swiss Institute of Allergy and Asthma Research, Davos, Switzerland) and Dr. Gerald Walter (Max Planck Institute of Molecular Genetics, Berlin, Germany, EU) in 2002. From 2002 to 2006 he developed CAGE (5'-SAGE) technology for large scale high throughput gene promoter mapping at RIKEN institute in Japan (with Dr. Piero Carninci and Prof. Yoshihide Hayashizaki). From 2006 to 2009 he worked in the laboratory of Nobel Prize Winner Prof. Sydney Brenner and Prof. Byrappa Venkatesh at the Institute of Molecular and Cell Biology in Singapore on creating libraries for the comparative Genomics project on Elephant shark. From 2009 he joined King Abdullah University of Science and Technology (KAUST) and spent one year in Joint Hong Kong University of Science and Technology (HKUST)/KAUST Micro/Nano-Fluidics Laboratory lead by Prof. Weijia Wen. Since 2010 he is working at KAUST in Saudi Arabia. His research interests are in the area of molecular biology, Genomics, Transcriptomics, surface chemistry, microfluidic systems and BioMEMS.

Xin Yi received his BS degree in physics from the Beijing Normal University, China, 2007. In 2008, he joined the Nano Science and Technology program, HKUST, Hong Kong and received M.Phil. degree in 2011. His main research areas are microfluidics, LOC (Lab-On-a-Chip), and BioMEMS.

Shunbo Li received his BS degree in Physics from Chongqing University, Chongqing, China, in 2009. Then he joined the Hong Kong University of Science and Technology, Hong Kong, China, where he is currently a Ph.D. student. His research interest is mainly focused on Micro/Nanofluidics, Micro/Nano-fabrication for Microdevices and functional electromagnetic materials.

Yu Sanna Hui received her B. Eng (2000) and M. Eng degrees (2004) at McGill University, Canada. She joined the PhD program in Nano Science and Technology Program at the Hong Kong University of Science and Technology in 2011. Her main research areas are fabrication of microfluidic devices and microspheres.

Weijia Wen earned his BS (1982) and MS (1988) degrees at Chongqing University. He completed his PhD (1995) degree in Institute of Physics, Chinese Academy of Science, Beijing. He was a postdoctoral fellow at HKUST (1995-1997) and UCLA (California University at Los Angeles) (1997-1999). He joined HKUST in 1999 and currently is a professor in the department of Physics, HKUST. Professor Wen's main research interests include soft condensed matter physics, electrorheological (ER) and magnetorheological (MR) fluids, field-induced pattern and structure transitions, micro- and nano-fluidic controlling, microsphere and nanoparticle fabrications, thin film physics, band gap materials, metamaterials and nonlinear optical materials. 